\title{
Michael Psellos im „Timarion“.
}

Dafs Michael Psellos in Timarions Hadesfahrt erwähnt wird, ist bekannt. Nur der Umfang dieser Erwähnungen dürfte zweifelhaft sein. Merkwürdig erscheint der Umstand, dafs der Name des Mannes nirgends genannt ist; er wird (Kap. 41) einfach als „der Sophist von Byzanz" eingeführt. Aber da wo dieser, bei dem unterirdischen Gerichtshof seit geraumer Zeit als Schreiber thätig, wegen seiner Raschheit und

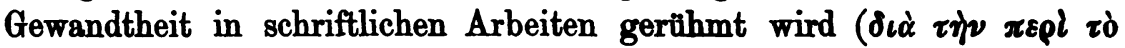

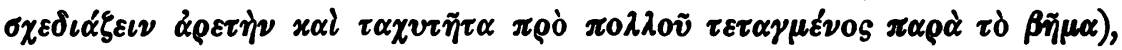
ist aus der näheren Kennzeichnung seiner Eigenart, beim Vorsprechen

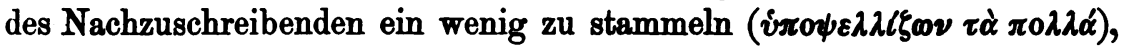
was, wie Timarion bemerkt, von seiner krummen Lippe herrühren mochte, die er noch nicht verloren hatte, mit Recht auf seinen Namen Psellos geschlossen worden. ${ }^{1}$ ) Dafs Psellos und kein andrer ferner unter "dem Sophisten von Byzanz" gemeint ist, läfst wenige Seiten später (Kap. 45) der Umstand erkennen, dafs dieser von den Sophisten ob der „Anmut und Lieblichkeit seines Vortrags, der lichtvollen Fafslichkeit seiner Rede, der leichten Gewandtheit des Ausdrucks und der Geschicklichkeit, womit er sie jedem Gegenstande anzupassen wufste", mit ausgezeichneter Ehrerbietung empfangen und jener stark therschwengliche Anfang einer an Kaiser Konstantinos Monomachos gorichteten Rede „O Herrschersonne!" (" $\Omega \beta \alpha \sigma \iota \lambda \varepsilon \tilde{v} \ddot{\eta} \lambda \iota \varepsilon)$, die wir jetzt noch unter den Werken des Psellos lesen ${ }^{2}$ ), wiederholt ihm zugerufen wird. Beweist die ehrende Bezeichnung "der Sophist von Byzanz" in einem etwa sechzig Jahre nach Psellos' Tode verfafsten Schriftwerke nicht mehr als alles andre, welchen Ruhm der vielgewandte Mann als Schriftsteller genofs ${ }^{3}$ ), sodals der aus Kappadocien stammende Verfasser des „Timarion“, hiefs er nun selbst Timarion oder anders, der durch seine ausgesprochene Liebe zur Philosophie sich selbst als einen Sophisten

1) A. Ellissen, Analekten der mittel- und neugriechischen Litteratur IV, S. 178, Anm. 118.

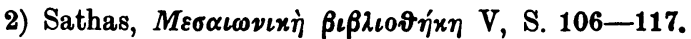

3) Vgl. meinen Aufsatz „Zu Michael Psellos" in Hilgenfelds Zeitschr. f. wiss. Theol. XXXII, S. 303-330, besonders S. 327-329. 


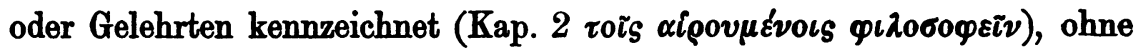
jede Nennung des Namens mit dem, was er in Verbindung mit jener Bezeichnung vorbrachte, seinen Zeitgenossen verständlich war?

Zu den beiden Stellen aus dem Schlufs des "Timarion" kommt aber meiner Überzeugung nach noch eine dritte, in der sich der: Schriftsteller ganz ähnlich wie dort verhält. Bei seiner traurigen Wanderung durch das Schattenreich stölst Timarion mit seinen Führern auf ein durch grelles Lampenlicht erhelltes, glänzend weifses Zelt, aus welchem laute Seufzer hervordringen. Während die Führer sich in Gespräche mit anderen Toten vertiefen, schaut Timarion verstohlen durch eine Öffnung in das Zelt, um zu sehen, was da vorgehe und woher der herzbrechende Jammer rühre. „Da sah ich“, erzählt er (Kap. 20, zumeist nach Ellissen), neinen Mann auf dem Boden liegen, dem die Augen ausgestochen waren ( der linken Seite und stätzte sich mit dem Ellenbogen auf einen lakonischen Teppich. Er war von hohem Wuchs, dabei ziemlich mager, aber starkknochig und von breiter Brust.... Bei ihm safs ein alter Mann, der mit Trostworten und Ermahnungen die mafslose Qual seines Flends zu lindern suchte. Der andere schien aber nicht darauf hören zu wollen, indem er öfters den Kopf schüttelte und den Alten mit der Hand fortstiefs. Es träufelte ihm auch Gift aus dem Munde." Auf seine Frage nach der Bedeutung dieses grausigen Zeltes und seiner $\mathrm{Be}-$ wohner erhält Timarion von einem der Toten folgende Auskunft (Kap. 22): „Der Bewohner dieses Zeltes, dessen tief schmerzdiches Gestöhn du vernommen, ist der berühmte Diogenes von Kappadocien. Seine übrigen Schicksale wirst du im Leben schon erfahren haben, wie er. zur kaiserlichen Würde gelangte, dann gegen die morgenländischen Soythen zu Folde zog und in ihre Gefangenschaft geriet. Da er nachber wieder frei kam und nach Byzanz zurlickkehrte, konnte er nicht wieder zur kaiserlichen Herrschaft gelangen, sondern im Kriege und überdies durch eine eidliche Versicherung abermals gefangen, wurde er treuloserweise, wio du siehst, geblendet, und damit nicht zufrieden, räumte man ihn noch tückisch mit verzehrendem Gift aus dem Wege. Der Alte aber, der bei ihm sitzt, stammt aus einem vornehmen Geschlechte in Grofs-Phrygien und diente ihm im Leben als Ratgeber und Gehilfe. Auch jetzt, voll Mitleids über des Diogenes Jammergeschick und des alten Verkehrs eingedenk, hält er beständig bei ihm aus und sucht das Gefuhl seiner herben Leiden durch angemessene Trost- und Vernunftgründe nach besten Kräften zu lindern." Gemeint ist Kaiser Romanos Diogenes (1067-1071); „an welchem" - nach Ellissens richtiger Bemerkung (a. a. O.S. 165, Anm. 75) - „Timarion, als des unglücklichen 
Fürsten kappadocischer Landsmann, wärmeren Anteil nehmen mochte". Das furchtbare Geschick desselben ist von Timarion in Übereinstimmung mit Bryennios und besonders Skylitzes kurz und ergreifend geschildert. Insbesondere wird Skylitzes' Angabe (bei Kedrenos II, 704) - Zonaras XVII, 15 ist wohl nur von jenem abhängig -, der Cäsar Johannes Dukas, des gestürzten und gefangenen Kaisers erbitterter Gegner, habe diesem, ehe er ihm auf unmenschliche, seinen qualvollen Tod herbeiführende Weise die Augen ausstechen liefs, Gift beigebracht, durch Timarion bestätigt.

Wer aber ist der Alte, der bei dem Kaiser sitzt? Hase vermutete, es könnte damit des Kaisers treuer Anhänger Kutatarios oder Chutaturios, der Herzog von Antiochia, gemeint sein. Denn dieser, nach Bryennios' Bericht ${ }^{1}$ ), ein aus Armenien gebürtiger, überaus tapferer und einsichtiger und von Diogenes bei seinem Regierungsantritt zu einer der höchsten Würden des Reichs beförderter Mann, war es - der komnenisch gesinnte Bryennios wagt das Ding nicht beim rechten Namen zu nennen -, der, nur dem Gefühle der Dankbarkeit folgend, zu seinem im tiefsten Unglück sitzenden Gebieter kam, ihm sichere Zuflucht in Cilicien anbot, ihm die festen Plätze des Landes überantwortete, ihm Soldaten, Geld, Waffen gab und ihn in eine Lage versetzte, dafs er an nahen Sieg zu glauben berechtigt war. „Doch dürtte", wie schon Ellissen (a. a. 0 . S. 165, Anm. 77) bemerkte, "dieser Annahme die ausdrückliche Bezeichnung des letzteren als eines Armeniers entgegenstehen." Dazu kommt, dafs Chutaturios des von ihm treu unterstützten Kaisers Geschick nicht geteilt hat, dafs er also auch mit diesem zu der Zeit, wo er, gräßslich verstümmelt, einem martervollen Tode entgegensah, nicht zusammengebracht werden kann. „Von Michsel Psellus“, fährt Ellissen fort, „den Hase bei dieser Gelegenheit auch nennt, jedoch nur, um der Vermutung auf ihn durch die Hinweisung auf die dem Romanus von ihm vielmehr bewiesene Feindseligkeit zu begegnen, kann um so weniger die Rede sein, da demselben, wie wir sehen werden, eine ganz andere Rolle in dieser Erzählung vorbehalten ist." Ich halte diese Begründung

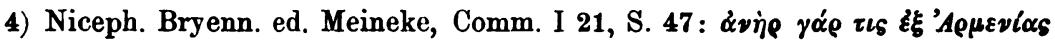

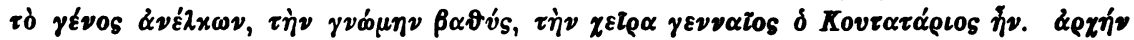

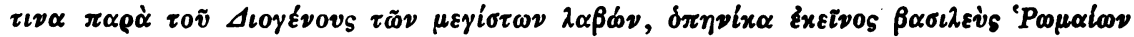

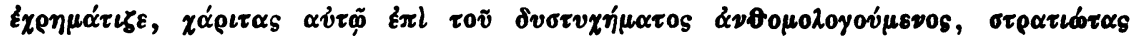

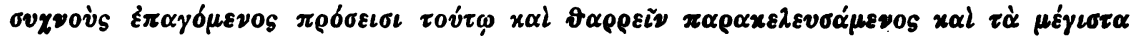

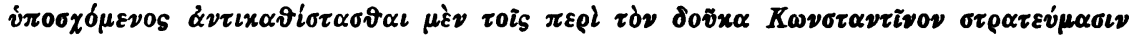

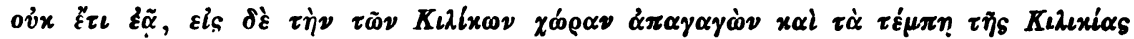

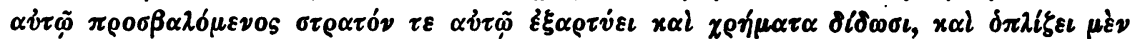

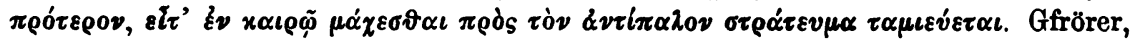
Byzantinische Geschichten III, S. 832/833. 
Hases und Elllissens für unzureichend und sehe in dem Alten vielmehr keinen anderen als Michael Psellos.

Zunächst empfiehlt es sich wenig, mit Ellissen von einer letzterem im "Timarion" vorbehaltenen besonderen Rolle zu reden. Psellos hat im Schattenreiche volle Bewegungsfreiheit. Einmal erscheint er als ein

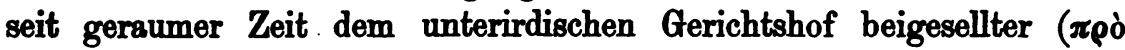

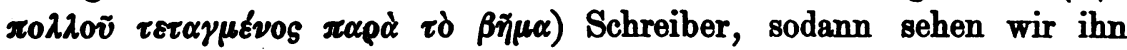
wieder frei herumwandeln und treffen ihn, wie er im Kreise der Sophisten ehrenvoll aufgenommen wird. Warum soll er nicht zu andrer Zeit bei dem bedauernswürdigen Kaiser Romanos Diogenes gesessen und diesem Trost zugesprochen haben? Dieser Zug nämlich ist es, der nach meiner Überzeugung nur auf Psellos pafst. Dals der Alte im "Timarion" a. a. 0. als Abkömmling eines edlen Geschlechtes aus GrofsPhrygien (

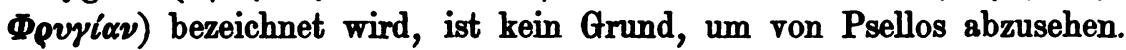
Denn dafs Psellos aus Byzanz stammte, wie Rhodius ${ }^{1}$ ) jüngst noch wieder behauptete, ist eine durch nichts genügend begröndete Annahme. Schwanken in den Nachrichten über die Herkunftsverhältnisse hervorragender Byzantiner kann man auch sonst bemerken. Um ein mir zur Hand liegendes Beispiel zu erwähnen, verweise ich auf den um mehrere Jahrhunderte jüngeren Gennadios Scholarios. Der Chier Leonardos und

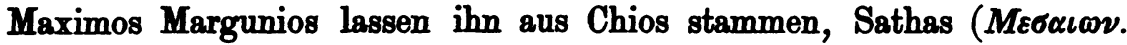
$\beta \iota \beta \lambda \iota 0$. VII, S. V) bezeichnet ihn als Thessaler, während er selbst in dem jüngst von A. Jahn herausgegebenen Dialog (vgl. Byz. Z. IV, S. 579, Anm. 1) Byzanz seine Vaterstadt nennt, in welche einst sein Vater aus Thessalien abersiedelte. Psellos' Zeitgenosse Michael aus Attalia, der, wie Seeger in seiner Besprechung der eben genannten Abhandlung von Rhodius (Byz. Z. II, 8. 149/150) den Bedenken Sathas' gegenuber nachwies, den berthmmten Gelehrten an drei Stellen seines Geschichtswerkes erwähnt, bezeichnet ihn (p. 296, 20) als aus Nikomedia stammend ( $\tau$ ò

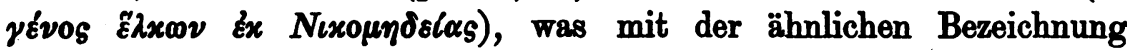
Scholarios' als Thessaler verglichen werden könnte. Ein Widerspruch mit der auf Byzanz als Heimat des Psellos weisenden Nachricht, wie ihn Rhodius in dem 135. Briefe (Sathas V, S. 378) zu finden meinte,

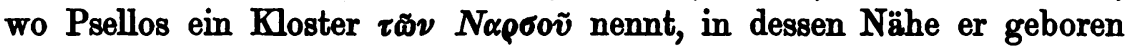

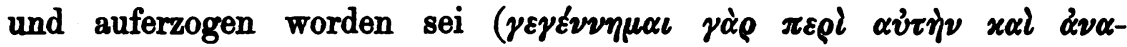
$\tau \dot{\varepsilon} \theta \rho \alpha \mu \mu \alpha \iota$ हैv $\alpha \dot{v} \tau \tilde{\eta})$, ist, wie Seeger ihm schon vorhielt, nicht vorhanden. „Wir wissen nicht", sagt derselbe, „wo dieses Kloster lag; bei Byzanz schwerlich, da es sonst wohl öfter erwähnt würde; warum

1) Bruno Rhodius, Boiträge zur Lebensgeschichte und zu den Briefen des Psellos (Plauen i. V. 1892. Progr. Nr. 541), S. 1. 
also nicht bei Nikomedia?" Im Hinblick auf die Stelle des "Timarion" frage ich ebenso: Warum nicht in Grofs-Phrygien, da der. Mangel an Erwähnung des Klosters, wie von Byzanz, ebenso auch von dem in der Geschichte jener Zeit so oft genannten Nikomedia hinwegweisen würde? Doch der Versuch, für Psellos' Herkunftsverhältnisse neue Angaben festzulegen, mufs so lange in der Luft schweben, als ich nicht zeigen kann, dafs die übrigen Angaben Timarions nur auf Psellos passen.

Hase lehnt die Beziehung Timarions auf Psellos mit Hinweis auf die dem Kaiser von ihm bewiesene Feindseligkeit ab. Man beachte, dafs dies ein Urteil ist, wie wir es eben heutzutage infolge tieferen Erfassens und Verstehens der Quellenschriften jener Zeit abzugeben vermögen. War Timarion in gleicher Lage? Ich bezweifle das. Bei der unverhohlenen Bewunderung, die er der wissenschaftlichen $\mathrm{Be}$ deutung des Psellos entgegenbringt, ist kaum anzunehmen, dafs er dem

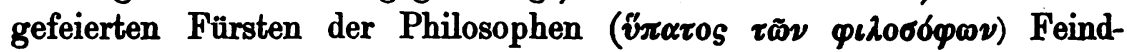
seligkeit gegen seinen wackeren, kriegsgewaltigen kappadocischen Landsmann, den unglücklichen Kaiser Romanos Diogenes, zugetraut hat, zumal wenn er, wie nicht zu bezweifeln, Psellos' Geschichtswerk gekannt hat. Aus dieser Kenntnis allein erklärt sich vollständig die Angabe, dafs der Alte, der jetzt, des früheren Verkehrs eingedenk ( $x \alpha \tau \dot{\alpha} \mu \nu \eta j \mu \eta \nu$

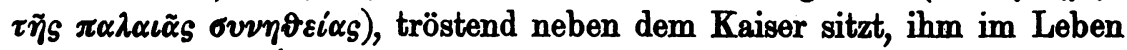

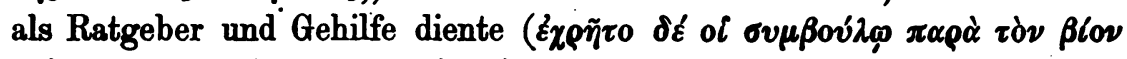

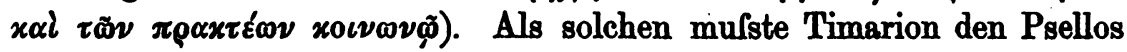
aus seinem Geschichtswerke kennen.

$\mathrm{Da}$ berichtet Psellos, wie entgegenkommend Romanos Diogenes sich $\mathrm{zu}$ ihm verhalten, wie er ihn geliebt und geehrt, wie er bei seinem Nahen vor ihm aufgestanden sei und ihm seine Zuneigung bewiesen habe. ${ }^{1}$ ) Psellos hebt ferner hervor, wie er, der keine andere Pflicht gekannt, als Kaisern nützliche Ratschläge zu geben, es sich habe angelegen sein lassen, die kriegerischen Neigungen des Kaisers durch den Hinweis auf die Schwierigkeit der Beschaffung der erforderlichen Streitkräfte und die Notwendigkeit der Anwerbung von befreundeten Söldnerscharen, kurz auf die Wichtigkeit einer in jeder Hinsicht ausreichenden Kriegsbereitschaft ein wenig zu hemmen. ${ }^{2}$ ) Er ruft Gott zum Zeugen

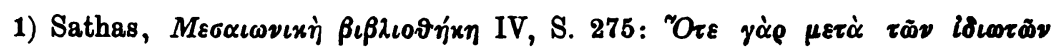

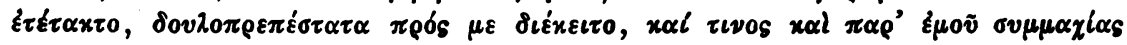

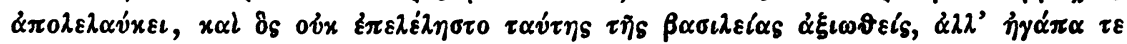

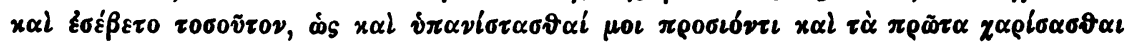

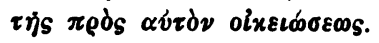

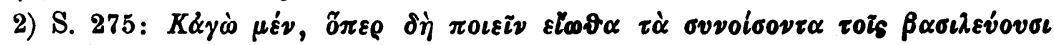

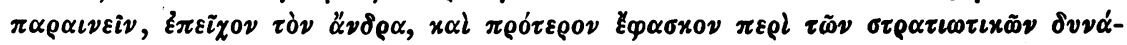


an, in welcher Sorge um die Hauptstadt und den Bestand des Reiches er des Kaisers Kriegspläne sich habe verwirklichen sehen. ${ }^{1}$ ) Der Pflicht, den Kaiser in den Feldzug zu begleiten, konnte er, wie wir nur aus dieser einzigen Stelle des Psellischen Geschichtwerks wissen, sich nicht entriehen. ${ }^{2}$ ) Der Kaiser wünschte, wie Psellos angiebt, von dessen strategischen Kenntnissen Vorteil zu ziehen; doch scheint sich der Ausgleich zwischen Wissenschaft und Leben, was nicht zu verwundern, nicht ohne eine gewisse Reibung vollzogen zu haben. ${ }^{3}$ ) Gleichwohl stellt sich Psellos beim Abwägen der kriegerischen Leistungen des Kaisers auf die Seite seiner Bewunderer (S. 279: $\varepsilon \gamma^{\prime} \dot{\omega}$ dz $\mu \varepsilon \tau \grave{\alpha} \tau \tilde{\tau} \boldsymbol{\nu}$

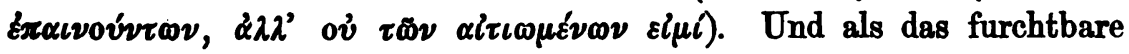
Geschick uber jenen von Seiten seines unversöhnlichen Gegners, des Cäsars Johannes Dukas, hereinbricht, ohne dafs, wie er versichert, er selbst oder die Umgebung der Kaiserin Eudokia, vor allen der an Stelle des gestürzten ausgerufene Kaiser Michael, Psellos' Zögling, etwas davon

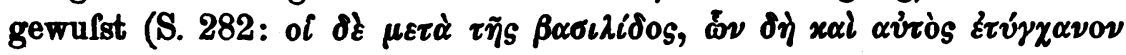

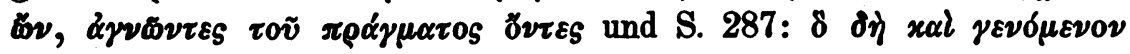
ทे vermelden, von denen er seufzend bekennt, dals sie besser ungeschehen

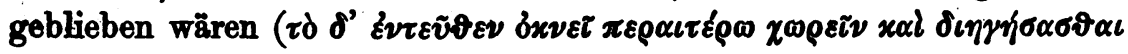

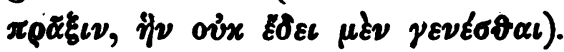

Mufste dies alles nicht dem wenige Menschenalter nach Psellos schreibenden Verfasser auf Grund von dessen Geschichtswerk die Überzeugung erwecken und bestärken, der von ihm als Philosoph und Redektunstler so hoch geschätzte Psellos sei Kaiser Romanos' Freund und treuer Berater gewesen? Doch wie er den Sophisten von Byzanz, der

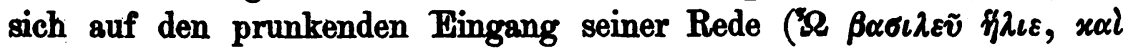

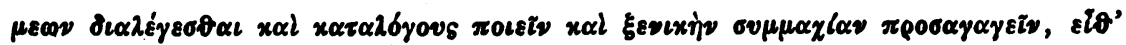

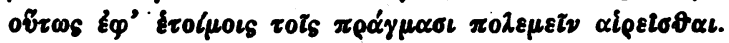

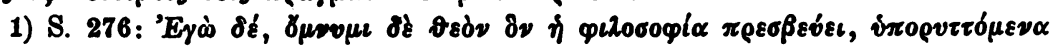

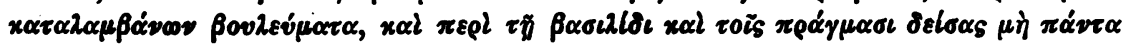

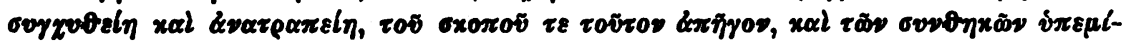

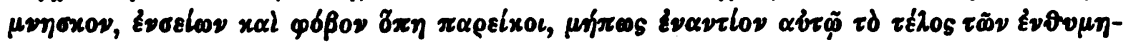

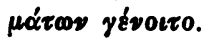

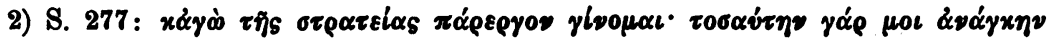

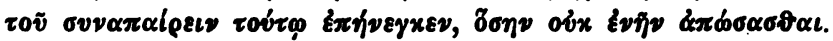

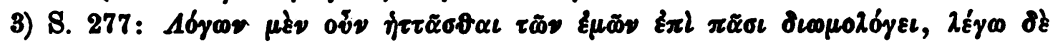

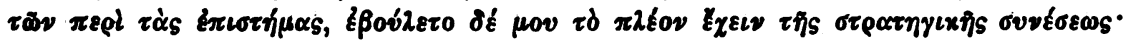

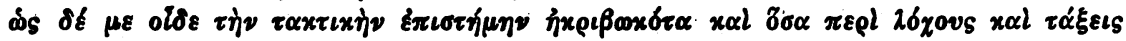

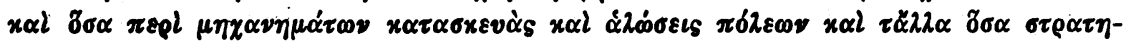

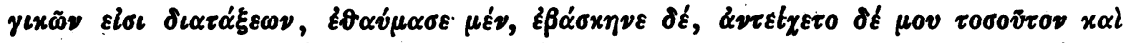

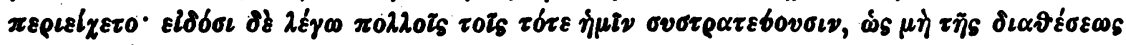

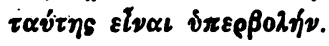




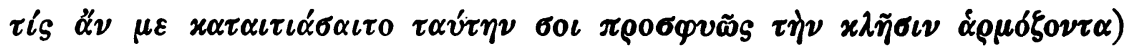
etwas zu gute that, ohne doch von der Sorge ganz frei zu sein, selbst in Byzanz dadurch einigen Anstofs zu erregen, durch Erwähnung desselben von Seiten der abgeschiedenen Sophisten nicht ganz ohne Spott lärst, so verfährt er auch bei der Kennzeichnung der Trostreden desselben.

Der Erklärung der Stelle würden wir beiläufig nicht gerecht werden, wenn wir an ein wirkliches Sitzen des Psellos neben dem vor Schmerzen stöhnenden Kaiser bei Lebzeiten denken wollten. Des Verfassers dichtender Geist schuf sich die Lagen und Verhältnisse für diese seine unterirdische Wanderung so, wie sie seinen Zwecken und der Wirkung entsprachen, die er zu erzielen beabsichtigte. Zu letzterer genügte vollkommen die Thatsache, dafs Psellos dem geblendeten Kaiser Romanos Diogenes Trost zu spenden versucht hat durch einen Brief. Dals Psellos' Briefe in abgeschlossener Sammlung frühzeitig allgemeiner bekannt geworden seien, ist kaum wahrscheinlich. Dafs das Schreiben desselben aber an den Unglücklichen weiteste Verbreitung finde, daran mufste der Regierung gelegen sein. Sollte dies doch vor allem auf die öffentliche Meinung wirken und. Kaiser Michael von dem Verdachte der Mitwissenschaft an der schmachrollen Blendung des gestürzten Herrschers reinigen. Hase und Ellissen waren mit den Briefen des Psellos, deren teilweise Herausgabe wir erst Sathas (1876) verdanken, nicht bekannt, sodafs sie gar nicht auf den Gedanken kommen konnten, jenes Trostschreiben zur Erklärung des „Timarion" heranzuziehen.

Und welches war denn der Trost, den Psellos spendete?

„Ich bin vollkommen ratlos, edelster und bewundernswertester $\mathrm{Mann}^{\prime}$ ", hebt er an (Brief 82, bei Sathas V, 316), „ob ich Dich als den allerunglücklichsten Menschen beweinen, oder als den ruhmvollsten Märtyrer bewundern soll. Denn wenn ich auf das Dir widerfahrene, alles Mafs übersteigende Leid blicke, so zähle ich Dich zu den Unglücklichsten, erwäge ich Deine schuldlose Gesinnung und Deinen Eifer für das Gute, so rechne ich Dich unter die Märtyrer, und wenn Du nach unzähligen Mifshandlungen noch hochherzig und dankbar gegen Gott bleibst, stelle ich Dich über die Märtyrer. Ich weifs nicht, ob je ein Mensch, und noch dazu ein in jeder Hinsicht unschuldiger, mit so schwerem Leid heimgesucht ward. Das aber vernimm aus meinem Munde, trefflichster Mann, alles, was da im Leben geschieht, hängt von der göttlichen Vorsehung und Leitung ab, und es giebt nichts, was vernunftwidrig, nichts, was nicht vorher beducht wäre. Auf alles schaut das nie schlummernde Auge Gottes hernieder, der den Duldern alle Mühsal und alles Mifsgeschick dieses Erdenlebens mit reichem Lohne 
vergilt. Ich weifs, es ist hart, des Augenlichtes beraubt zu werden, und zwar um so härter, als jenes mit voller Absicht den Schlufs vieler vorhergegangener Mifshandlungen bildete; aber ich weifs andrerseits auch, dafs es etwas Grofses ist, des göttlichen Lichtes zu geniefsen .... Gieb Gott die Ehre, dals er Dich, der Du ein Mensch bist, zum Engel machte und Dich, der $\mathrm{Du}$ der Augen beraubt bist, eines herrlicheren Lichtes für würdig erachtete, Dich in die Reihen seiner edlen Kämpfer stellte und, nachdem er die irdische Krone Dir genommen, Dich mit himmlischem Kranze schmückte ... Leuchtend wirst Du zur Rechten des Richters gestellt werden, glänzend gekrönt mit dem Diadem der Märtyrer, mit geöffneten Augen die wunderbaren Geheimnisse der Gottheit schauend. Märtyrer werden Deine schmerzenden Augen küssen, Engel werden, ja kühnlich sage ich es, Gott selbst Dich herzen."

Sind das angemessene, nach besten Kräften gespendete Trost- und

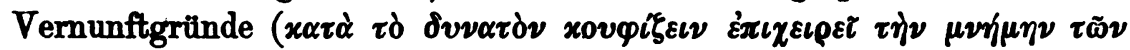

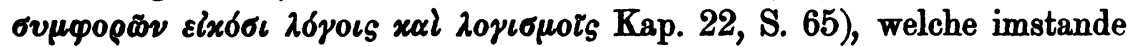
gewesen wären, die malslose Qual des kaiserlichen Dulders ( $\tau$ ì $\tau \tilde{\eta} s$

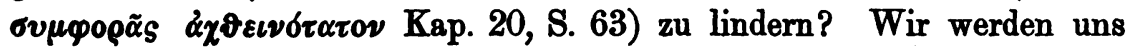
nicht wundern dürfen, wenn es Timarion so schien, als ob der Unglück-

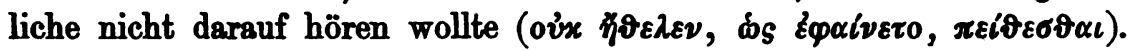

Und wenn der Alte nun gar fortfährt: „Vor allem schwöre ich Dir bei dem wahrhaftigen Gott, dafs der Kaiser an alledem, was Dir widerfahren, in jeder Hinsicht unschuldig ist... Schmerz erfüllte seine Seele, als er davon hörte, seufzend vergofs er Ströme von Thränen, oftmals wünschte er sich den Tod, wünschte, dafs die Erde ihn verschlingen möchte: o glaube mir doch, ich rede die lautere Wahrheit, noch hat er sich nicht trösten lassen, sondern sagt sich rom Leben selbst los" -: dann verstehen wir es, wenn der Kaiser, der auch nach Psellos' Zeugnis (s. o.) seine Leiden mit der Ergebenheit eines Christen trug, sein - wie Skylitzes (bei Kedrenos II, 704) es beschreibt - bis zur Unkenntlichkeit angeschwollenes Haupt, dessen wunde Augenhöhlen eiternd in Fäulnis übergingen, nach Timarions Bericht öfters zweifelnd

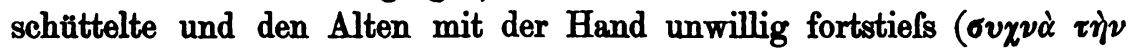

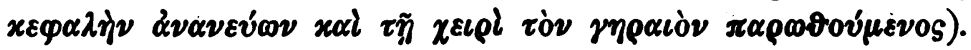

Das war der aalglatte Höfling Michael Psellos, der Sophist von Byzanz, der Furst unter den Philosophen. Ja wahrlich, Timarion that recht daran, dafs er jene Rolle desselben für die Schilderung der Schrecken der Unterwelt sich nicht entgehen liefs.

Wandsbeck.

Johannes Dräseke. 